\title{
EVALUACIÓN DE LA ESCALA MORISKY DE ADHERENCIA A LA MEDICACIÓN (MMAS-8) EN ADULTOS MAYORES DE UN CENTRO DE ATENCIÓN PRIMARIA EN CHILE
}

\author{
Fernando Valencia-Monsalvez ${ }^{1, a}$, Sara Mendoza-Parra ${ }^{1, b}$, Luis Luengo-Machuca ${ }^{2, c}$
}

\begin{abstract}
RESUMEN
Con el objetivo de determinar si la escala de adherencia a la medicación de Morisky de 8 ítems (MMAS-8) es un instrumento útil en adultos mayores hipertensos que acuden a un centro de atención primaria, se realizó un estudio de campo con abordaje cuantitativo de evaluación de pruebas diagnósticas, en ancianos hipertensos del Programa de Salud Cardiovascular de Cabrero, Chile. Los resultados de la escala fueron comparados con la presión arterial y el método de recuento de comprimidos. La evaluación diagnóstica de la escala utilizando los métodos de referencia presión arterial y recuento de comprimidos dio como resultado: sensibilidad: $86 \%$, $85 \%$; especificidad: $56 \%, 58 \%$; valor predictivo positivo: $60 \%, 65 \%$; valor predictivo negativo: $84 \%$. $81 \%$. En conclusión, la escala permite detectar de forma simple, y a bajo costo, a personas que no son adherentes a su régimen de tratamiento medicamentoso.
\end{abstract}

Palabras clave: Valor predictivo de las pruebas, Cumplimiento de la medicación, Adulto Mayor (fuente: DeCS BIREME).

\section{EVALUATION OF MORISKY MEDICATION ADHERENCE SCALE (MMAS-8) IN OLDER ADULTS OF A PRIMARY HEALTH CARE CENTER IN CHILE}

\begin{abstract}
In order to determine whether the Morisky 8-item medication adherence (MMAS-8) is a useful instrument in elderly hypertensive patients attending a primary care center, we conducted a field study with a quantitative approach of evaluation of diagnostic tests in elderly hypertensive patients from the Cardiovascular Health Program Health in Cabrero, Chile. The results of the scale were compared with the blood pressure and the pill-count method. The diagnostic evaluation of the scale using the reference methods of blood pressure and pill count resulted: Sensitivity: $86 \%$, $85 \%$; specificity: $56 \%$, $58 \%$; positive predictive value: $60 \%, 65 \%$; negative predictive value: $84 \% .81 \%$. In conclusion, the scale allows to detect in a simple way, and at a low cost, to people who are not adherent to their regimen of drug treatment.
\end{abstract}

Key words: Predictive Value of Tests, Medication adherence, aged (source: MeSH NLM).

\section{INTRODUCCIÓN}

Las enfermedades del aparato circulatorio constituyen la primera causa de muerte en los países en desarrollo intermedio o avanzado, como consecuencia de la transición epidemiológica, causando graves problemas sanitarios, a pesar de que se pueden disminuir si se controlan sus factores de riesgo ${ }^{(1)}$ tales como el tabaquismo, diabetes mellitus, niveles elevados de colesterol, sobrepeso u obesidad e hipertensión arterial (2). Esta última es causa, por lo menos, del $45 \%$ de las muertes por cardiopatías y el $51 \%$ de las muertes por accidentes cerebrovasculares ${ }^{(3)}$.
Debido a la alta prevalencia de la hipertensión arterial y su morbilidad asociada, su control constituye un importante reto para la salud pública. Este control es pobre pese a la existencia de fármacos antihipertensivos y de la implementación de diversas estrategias no farmacológicas dirigidas a la modificación de estilos de vida ${ }^{(4)}$.

La Organización Mundial de la Salud (OMS) declara que la adherencia deficiente a los tratamientos a largo plazo compromete gravemente la efectividad de los tratamientos ${ }^{(5)}$, especialmente en quienes tienen una mayor probabilidad de protagonizar una conducta

Facultad de Enfermería, Universidad de Concepción. Concepción, Región del Bío Bío, Chile.

Facultad de Odontología, Universidad de Concepción. Concepción, Región del Bío Bío, Chile.

Enfermero. magíster en Enfermería; ${ }^{\mathrm{b}}$ enfermera. doctora en Enfermería; ${ }^{\mathrm{c}}$ licenciado en Educación con Mención en Matemáticas y Física, Magíster en Estadística Aplicada.

Recibido: 08/06/2016 Aprobado: 24/05/2017 En línea: 28/06/2017

Citar como: Valencia-Monsalvez F, Mendoza-Parra S, Luengo-Machuca L. Evaluación de la escala Morisky de adherencia a la medicación (MMAS-8) En adultos mayores de un centro de atencion primaria en Chile. Rev Peru Med Exp Salud Publica. 2017;34(2):245-9. doi: 10.17843/rpmesp.2017.342.2206 
incumplidora a lo largo del tiempo ${ }^{(6)}$, por lo que este estudio se fundamenta en la necesidad de contar con instrumentos sensibles y aplicables en diferentes situaciones rápida y económicamente, que permitan medir adecuadamente esta conducta, y así gestionar intervenciones diferenciadas, precisas y focalizadas, reduciendo la morbimortalidad prematura en este grupo de personas. En este sentido, la escala de adherencia a la medicación de Morisky ha sido utilizada frecuentemente en investigaciones sobre la adherencia al tratamiento antihipertensivo, pero en Chile no ha sido sometidas a evaluación diagnóstica.

Por lo anterior, el objetivo de este estudio es determinar si la escala de adherencia a la medicación de Morisky ocho ítems (MMAS-8) es un instrumento indirecto útil para medir la conducta de adherencia terapéutica en adultos mayores hipertensos en tratamiento en atención primaria, confrontando sus resultados con los métodos de comparación de presión arterial y recuento de comprimidos.

\section{MATERIALES Y MÉTODOS}

Estudio de campo con abordaje cuantitativo cuyo diseño fue de evaluación de pruebas diagnósticas utilizando como unidad de análisis a pacientes hipertensos adultos mayores (65 años o más) ingresados al Programa Cardiovascular de Atención Primaria de Cabrero, VIII Región, Chile.

Para determinar el tamaño de la muestra inicialmente se pensó hacer un muestreo probabilístico aleatorio simple con un $95 \%$ de confiabilidad de la población total de dichos pacientes (1284), pero al aplicar los criterios de inclusión y exclusión al universo se obtuvieron 330 personas. Frente a esto se optó por trabajar con la población y no con una muestra.

Los criterios de inclusión fueron: adulto mayor con hipertensión arterial, sin otras patologías crónicas diagnosticadas, verificado a través de la revisión de las fichas clínicas en el centro de salud; tener al menos 6 meses de antigüedad en el programa cardiovascular, indicación médica de consumo de fármacos antihipertensivos orales y consentir su participación en el estudio. Fueron excluidos pacientes con otras enfermedades crónicas asociadas, evidencias de discapacidad mental o física que impidieran seguir un tratamiento, personas institucionalizadas y postradas.

La recolección de los datos se aplicó en el domicilio de los pacientes, la escala MMAS-8 y los métodos de comparación "toma estandarizada de la presión arterial" y "método de recuento de comprimidos" descritos a continuación:

Escala de adherencia a la medicación de Morisky 8 ítems (MMAS-8) (Tabla 1): mide comportamientos

\section{MENSAJES CLAVE}

Motivación para realizar el estudio. El control de la hipertensión arterial y su morbilidad asociada constituye un importante reto para la salud pública en este sentido, la escala de adherencia a la medicación de Morisky ha sido utilizada frecuentemente en investigaciones sobre la adherencia al tratamiento antihipertensivo, pero en Chile no ha sido sometidas a evaluación diagnóstica.

Principales hallazgos. La evaluación diagnóstica de la escala Morisky utilizando los métodos de comparación presión arterial y método de recuento de comprimidos, dieron como resultado: sensibilidad: $86 \%, 85 \%$; especificidad: $56 \%, 58 \%$; valor predictivo positivo: $60 \%, 65 \%$; valor predictivo negativo: $84 \%, 81 \%$.

Implicancias. La escala de adherencia a la medicación de Morisky 8 ítem (MMAS-8) es un instrumento sensible para detectar a los pacientes que no cumplen apropiadamente el régimen terapéutico.

de adherencia específicos asociados a la ingesta de medicamentos, con siete preguntas cuyas alternativas de respuestas son Sí/NO, y una pregunta en escala de tipo Likert. Ha sido validada en pacientes hipertensos ${ }^{(7,8,9,10)}$. La adherencia se determinó de acuerdo a su puntación, clasificando como adherentes a los pacientes con 8 puntos y no adherentes con puntajes menores a 8. La consistencia interna de esta escala cuantificada con alfa de Cronbach fue de 0,646 .

Toma de presión estandarizada: se utilizó esto, puesto que clasifica a los pacientes de acuerdo a su presión arterial, es decir, normal ( $<140 / 90 \mathrm{~mm} \mathrm{Hg}$ ) y alterada $(\geq 140 / 90 \mathrm{~mm} \mathrm{Hg}$ ) respectivamente, de acuerdo a lo establecido en la Guía Clínica de Hipertensión Arterial (11).

Recuento de comprimidos: es sencillo y objetivo ${ }^{(5,12)}$. Compara el número de comprimidos que quedan en el sobre, teniendo en cuenta los prescritos y el tiempo transcurrido entre la prescripción y el recuento. Se obtiene según la siguiente fórmula: $\left(\mathrm{N} .^{\circ}\right.$ total de comprimidos presumiblemente consumidos $/ \mathrm{N} .^{\circ}$ total de comprimidos que debía haber consumido) x 100 . Se considera que los límites de ese porcentaje, que definen el buen cumplimiento o adherencia, se sitúan en el consumo del $80-110 \%$ de los comprimidos prescritos. Valores inferiores a $80 \%$, o superiores a $110 \%$ expresan hipocumplimiento e hipercumplimiento, respectivamente (13). Para la evaluación diagnóstica fueron considerados adherentes a los que clasificaron como normocumplidores y como no adherentes a hiper e hipocumplidores. Se optó por utilizar este método debido a que la OMS ${ }^{(5)}$ lo recomienda para medir la adherencia en pacientes con enfermedades crónicas que deben consumir preparaciones farmacológicas sólidas.

Para analizar los datos se realizó descripción univariada de las variables, tanto sociodemográficas como de antecedentes de salud, usando tablas de frecuencias para variables cualitativas, medidas de resumen, 
Tabla 1. Escala de Adherencia a la Medicación de Morisky 8 ítems (MMAS-8)

\begin{tabular}{|c|c|c|}
\hline \multirow{2}{*}{$\begin{array}{l}\text { Preguntas } \\
\text { 1. ¿Se le olvida alguna vez tomar la medicina para su hipertensión arterial? }\end{array}$} & \multicolumn{2}{|c|}{ Opciones de respuesta } \\
\hline & Sí=0 & $\mathrm{No}=1$ \\
\hline $\begin{array}{l}\text { 2. A algunas personas se les pasa tomarse sus medicinas por otras razones y no } \\
\text { un simple olvido. Si recuerda las últimas dos semanas, ¿hubo algún día en el } \\
\text { que se le olvidó tomar la medicina para su hipertensión arterial? }\end{array}$ & Sí=0 & $\mathrm{No}=1$ \\
\hline $\begin{array}{l}3 \text { ¿Alguna vez ha reducido la dosis o directamente dejado de tomar la medicina } \\
\text { sin decírselo a su médico porque se sentía peor al tomarla? }\end{array}$ & Sí=0 & $\mathrm{No}=1$ \\
\hline $\begin{array}{l}\text { 4. Cuando viaja o está fuera del hogar, ¿se le olvida llevar la medicina para su } \\
\text { hipertensión arterial alguna vez? }\end{array}$ & Sí=0 & $\mathrm{No}=1$ \\
\hline *5. ¿Tomó la medicina para su hipertensión arterial ayer? & Sí=1 & $\mathrm{No}=0$ \\
\hline $\begin{array}{l}\text { 6. Cuando siente que su hipertensión arterial está bajo control, ¿deja a veces de } \\
\text { tomar su medicina? }\end{array}$ & Sí $=0$ & $\mathrm{No}=1$ \\
\hline $\begin{array}{l}\text { 7. Tomar medicamentos cada día puede ser un problema para muchas personas. } \\
\text { ¿Se siente alguna vez presionado por seguir el tratamiento médico para su } \\
\text { hipertensión arterial? }\end{array}$ & Sí $=0$ & $\mathrm{No}=1$ \\
\hline \multirow[t]{2}{*}{ 8. ¿Con qué frecuencia tiene dificultades para recordar tomar todas sus medicinas? } & $\begin{array}{c}\text { Nunca/Casi nunca } \\
\text { Rara vez } \\
\text { Algunas veces } \\
\text { Habitualmente }\end{array}$ & $\begin{array}{c}1 \\
0,75 \\
0,5 \\
0,25\end{array}$ \\
\hline & Siempre & 0 \\
\hline
\end{tabular}

Fuente: Morisky D, Ang A, Krousel-Wood M, Ward H. Predictive Validity of A Medication Adherence Measure in an Outpatient Setting. J Clin Hypertens. 2008; 10(5): 348-354 ${ }^{(7)}$.

*Inversión de puntaje

tendencia central y dispersión para las variables cuantitativas. En cuanto a las propiedades psicométricas de las escalas, se usó alfa de Chronbach para la consistencia interna. Para determinar asociación entre variables cualitativas se utilizó la prueba chi cuadrado. Para cuantificar la eficacia de las pruebas diagnósticas se utilizaron indicadores de sensibilidad, especificidad, valores predictivos positivo y negativo y cocientes de probabilidades, obteniendo la estimación puntual y sus respectivos intervalos de confianza. Los datos fueron analizados con los programas SPSS 12 y EPIDAT 3.1 . Los contrastes se consideraron significativos al $5 \%$.

La investigación fue evaluada por el Comité de Ética de la Facultad de Medicina de la Universidad de Concepción, Chile.

\section{RESULTADOS}

De la población analizada, 143 personas tuvieron la presión arterial elevada ( $43 \%$ de los encuestados). Los que clasificaron como no adherentes, con el método de recuento de comprimidos, fueron 157 (47,6\%); mientras que el $62,1 \%$ clasifican como no adherentes con la utilización de la escala MMAS-8. En este grupo de personas, la pregunta de la escala MMAS-8 que contribuyó mayormente a puntuar con "no adherencia" fue la pregunta 1 , que tiene relación con el olvido de tomar los medicamentos para el control de la presión arterial (32\% de los encuestados), seguida de la pregunta 2, que indaga sobre el haber olvidado ingerir medicamentos las últimas 2 semanas $(21 \%$ de los encuestados).

La evaluación diagnóstica de la escala utilizando los métodos de comparación presión arterial y método de recuento de comprimidos, dieron como resultado: sensibilidad: $86 \%, 85 \%$; especificidad: $56 \%, 58 \%$; valor predictivo positivo: $60 \%, 65 \%$; valor predictivo negativo: $84 \%, 81 \%$, coeficiente de cociente de probabilidades positivo 1,96 y 2,04; cociente de probabilidades negativo 0,25 y 0,26 respectivamente (Tabla 2 ).

Tabla 2. Valores diagnósticos obtenidos a partir de la utilización de escala de adherencia a la medicación de Morisky 8 ítems (MMAS-8) y los métodos de comparación: presión arterial y recuento de comprimidos

\begin{tabular}{lcccc}
\hline Valores diagnósticos & Presión arterial & (IC 95\%) & $\begin{array}{l}\text { Recuento de } \\
\text { comprimidos }\end{array}$ & (IC 95\%) \\
\hline Sensibilidad (S-MMAS-8) & 86 & $(79,9-92,1)$ & 85 & $(78,8-90,7)$ \\
Especificidad (E-MMAS-8) & 56 & $(48,8-63,5)$ & 58 & $(50,8-66,0)$ \\
Valor predictivo positivo (VPP-MMAS-8) & 60 & $(53,1-66,9)$ & 65 & $(58,1-71,7)$ \\
Valor predictivo negativo (VPN-MMAS-8) & 84 & $(77,2-90,8)$ & 81 & $(73,5-88,1)$ \\
Cociente de probabilidades positivo & 1,96 & $(1,7-2,3)$ & 2,04 & $(1,69-2,46)$ \\
Cociente de probabilidades negativo & 0,25 & $(0,2-0,4)$ & 0,26 & $(0,18-0,39)$ \\
\hline
\end{tabular}


Sobre el análisis bivariado de las variables, existe asociación estadísticamente significativa entre la clasificación de la escala MMAS-8 (adherente y no adherente) con la clasificación de la presión arterial (normal, alterada) $(p<0,0001)$.

\section{DISCUSIÓN}

El porcentaje de adultos mayores en control con valores de presión arterial $\geq 140 / 90 \mathrm{~mm} \mathrm{Hg}$, fue de $43 \%$, lo que es mayor a la descompensación alcanzada a nivel nacional (37\%) durante el año 2012 (14), pero cercano a la literatura internacional (Francia ${ }^{(9)} 39,5 \%$ y Brasil ${ }^{(10)} 34 \%$ ) utilizando la presión arterial como referencia. Lo anterior es comparable a los antecedentes que entregó la OMS ${ }^{(5)}$, ya que se espera que el $50 \%$ sea adherente y el restante no adherente. Este es un hallazgo importante porque la baja adherencia perjudica la evolución clínica de los pacientes, causando aumento de la morbimortalidad y de los gastos de salud.

La consistencia interna de la escala MMAS-8, medida por el alfa de Cronbach, fue 0,646 , inferior a la obtenida por Morisky et al. en su estudio con pacientes hipertensos $(0,83)^{(7)}$. El grupo de Morisky estaba constituido por 1367 personas que promediaban los 52,5 años, sobre el $50 \%$ graduados de enseñanza media. En Brasil (10), el alfa de Cronbach fue $(0,69)$ en una población de 223 , con un promedio de 57,2 años. En Francia ${ }^{(9)}$ fue 0,54 con una muestra de 199 pacientes, que promediaban los 55,7 años. En este último, Korb-Savoldelli fundamenta este resultado basándose en la diferencia poblacional con respecto al estudio original. La población de esta investigación, 330 pacientes, era en su totalidad adulta mayor que, en promedio, tenían 73,6 años, por lo que se presume que estos son factores que pueden influir en la diferencia del alfa de Cronbach con respecto al estudio original.

Los valores de sensibilidad y especificidad de la escala MMAS-8, indican que permite detectar a los pacientes que no son adherentes a seguir su tratamiento medicamentoso, mientras que su especificidad indica que es un instrumento que permite pesquisar moderadamente a los pacientes que, efectivamente, cumplen con las indicaciones relacionadas con la ingesta de medicamentos.

Los coeficientes de probabilidades describen a esta prueba, en donde la clasificación de los pacientes como "no adherentes" es dos veces más probable en pacientes descompensados que en pacientes compensados.

La asociación del puntaje de la MMAS-8 con la presión arterial, que también se presenta en estudios de similares características ${ }^{(7,9,10)}$, supone un resultado de gran importancia para los equipos de atención primaria, ya que se puede evidenciar que el uso efectivo de este instrumento en la práctica clínica, constituiría una herramienta que apoye la pesquisa oportuna de pacientes no adherentes, ya que se podría intervenir oportunamente a quienes no cumplan, minimizando los factores de riesgo para los pacientes hipertensos. De acuerdo a lo expuesto, esta escala se podría aplicar en las personas en estadios iniciales de ingreso al programa de salud cardiovascular, de tal forma de evaluar la adherencia desde un principio, y determinar el riesgo de no adherencia. Lo anterior permitiría focalizar mejor las intervenciones a los individuos no adherentes, evitando la morbimortalidad prematura.

Este estudio evidenció que el factor que mayormente se relacionó con la "no adherencia" fue el olvido, específicamente en las preguntas 1 y 8 . Se puede determinar, de acuerdo a esto, que olvidar tomar medicamentos es un factor que repercute negativamente en la salud de los adultos mayores hipertensos, dificultando su adherencia a largo plazo y aumentando la probabilidad de aparición de factores de riesgo. Si tenemos esta visión, todo lo que se planifique debe apuntar más apropiada y certeramente a acompañar al paciente, gestionando los cuidados considerando las características de cada persona que repercuten en la memoria. El olvido es una variable importante que provoca barreras para la ingesta medicamentosa y cuando avanza la edad de los pacientes, se pueden implementar diferentes estrategias para apoyarlos en la medida que vayan cumpliendo años, ya que no es lo mismo orientar a una persona de 60 años que puede recordar todo su tratamiento para la hipertensión, versus un octogenario, que tiene disminuidas sus capacidades de memorizar. Otras estrategias para asegurar la ingesta de medicamentos, pueden incluir llamados telefónicos, uso de dispensadores electrónicos de medicamentos, alarmas, etc. Por lo mencionado anteriormente el uso de esta escala que es sensible a pesquisar la no adherencia en los adultos mayores, debería ser complementada con instrumentos que permitan valorar la memoria, para focalizar de forma más eficaz las estrategias para aumentar la adherencia y el éxito de las terapias antihipertensivas.

En conclusión, la evaluación diagnóstica de la escala de Morisky de 8 ítems indica que es un instrumento sensible para detectar a los pacientes que no cumplen apropiadamente el régimen terapéutico y que es efectiva para estimar la probabilidad de que, detectado un adherente a seguir la medicación, verdaderamente sea adherente a la ingesta medicamentosa.

Fuentes de financiamiento: investigación realizada con el financiamiento del Fondo de Innovación Tecnológica de la Región del Bio- Bio “INNOVA BIO-BIO” Código: 12.369. 
Conflictos de interés: los autores no presentan conflictos de interés en la publicación del artículo.

Contribuciones de autoría: FVM participó en la concepción y diseño del artículo, recolección, análisis e interpretación de datos y obtención de fuentes de financiamiento. SMP participó en la concepción y diseño del artículo, análisis e interpretación de datos y obtención de fuentes de financiamiento. LLM participó en análisis e interpretación de datos. Todos los autores han dado su revisión crítica del artículo.

\section{REFERENCIAS BIBLIOGRÁFICAS}

1. Galve E, Cordero A, Bertomeu V, Fácila L, Mazón P, Alegría E, et al. Novedades en cardiología: riesgo vascular y rehabilitación cardiaca. Rev Esp Cardiol. 2015; 68(2):136-43.

2. Wong N. Epidemiological studies of $\mathrm{CHD}$ and the evolution of preventive cardiology. Nature Reviews Cardiology. 2014; 11, 276-289.

3. Organización Mundial de la Salud. [Internet] 2013. Información general sobre la hipertensión en el mundo. [acceso el 1 de Abril de 2016]. Disponible en: http://apps.who.int/iris/ bitstream/10665/87679/1/WHO_ DCO_WHD_2013.2_spa.pdf

4. Varela M. El reto de evaluar la adherencia al tratamiento en la hipertensión arterial. Rev Pensamiento Psicológico. 2010; 7(14): 127-140.

5. Organización Mundial de la Salud Adherencia a los tratamientos a largo plazo Pruebas para la acción. Washington, D.C.:OPS; 2004.

6. Mendoza S, Merino J, Barriga O. Identificación de factores de predicción del incumplimiento terapéutico en adultos mayores hipertensos de una comunidad del sur de Chile. Rev Panam Salud Pública. 2009 Feb; 25(2):105-120.

7. Morisky D, Ang A, Krousel-Wood M, Ward H. Predictive Validity of A Medication Adherence Measure in an Outpatient Setting. J Clin Hypertens. 2008; 10(5): 348-354.

8. Muntner P, Joyce C, Holt E, He J, Morisky D, Webber LS, Krousel-Wood M. Defining the Minimal Detectable Change in Scores on the Eight-Item Morisky Medication Adherence Scale. Ann Pharmacother. 2011;45:569-75.

9. Korb-Savoldelli V, Gillaizeau F, Pouchot J, Lenain E, Postel-Vinay $\mathrm{N}$, Plouin J, et al. Validation of a French Version of the 8-Item Morisky Medication Adherence Scale in Hypertensive Adults. J Clin Hypertens. 2012; 14:429-434.

10. Dias A, Barreto-Filho J, Felizardo S, Pereira D. Relação entre a Escala de Adesão Terapêutica de oito itens de Morisky (MMAS-8) e o Controle da Pressão Arterial. Arq Bras Cardiol. 2012; 99(1):649-658.

11. Ministerio de Salud. Guía Clínica Hipertensión Arterial Primaria o
Esencial en personas de 15 años y más. Series Guías Clínicas. Santiago: Minsal; 2010.

12. Márquez E. Estrategias para mejorar el cumplimiento terapéutico en la hipertensión arterial.[Internet] 2003 Sep [acceso el 25 de agosto 2013]. Disponible en: http://www.fac.org.ar/ tcvc/llave/c053/marquez.htm

13. Márquez Contreras E. Evaluación del incumplimiento en la práctica clínica. Hipertensión. 2008 Sep; 25(5):205-

14. Ministerio de Salud. Programa Nacional de salud de las personas adultas mayores. Santiago: Minsal; 2014.

Correspondencia: Fernando Antonio Valencia Monsalvez

Dirección: Facultad de Enfermeria, Universidad de Concepción, Chile, Calle Roosevelt 1787

Teléfono: 56984745648,

Correo electrónico: fernandovalencia.m@ gmail.com

\section{Nuestros artículos se encuentran indizados en:}

$$
\text { PublMed }
$$

